\title{
Adverse experiences in childhood influence brain responses to emotional stimuli in adult psychiatric patients
}

\author{
Katharina Matz ${ }^{\mathrm{a}, *}$, Markus Junghöfer ${ }^{b}$, Thomas Elbert ${ }^{a}$, Katja Weber ${ }^{a}$, \\ Christian Wienbruch ${ }^{a}$, Brigitte Rockstroh ${ }^{a}$ \\ a Department of Psychology, University of Konstan, Germany \\ ' Institute for Biomagnetism, University of Münster, Germany
}

\section{A R T I C L E I N F O}

Article history:

Received 7 September 2009

Received in revised form 1 December 2009

Accepted 16 December 2009

Available online 4 January 2010

\section{Keywords:}

Early life stress

Affective pictures

Mental disorder

Magnetoencephalography

RSVP

\begin{abstract}
A B S T R A C T
Previous results suggest that early life stress (ELS) may be related to altered cortical responses to emotional stimuli. In a previous study, we found suppressed cortical responses to emotional pictures in psychiatric patients with high-ELS. The present study explored the stability of this effect across time and stimulation conditions. In addition, the relationship between ELS and current life stress was examined, and we probed whether this current life stress was related to the cortical responses. Fifteen patients with high, 16 patients with low-ELS and 15 psychiatrically healthy subjects with low-EIS participated in two sessions 8 months apart. Subjects monitored a rapid serial presentation of pleasant, neutral and unpleasant pictures during magnetoencephalographic recording. In both sessions, estimated neural activity in occipital-parietal-temporal regions between 70 and $250 \mathrm{~ms}$ after picture onset was smaller in patients, particularly in those with high-EIS, compared to healthy subjects. Modulation of activity by arousing (pleasant and unpleasant) compared to neutral stimuli around $200 \mathrm{~ms}$ post-stimulus did not differ between groups, whereas around $300 \mathrm{~ms}$, patients did not show the pronounced cortical response to pleasant stimuli exhibited by healthy subjects. Results suggest that ELS and psychiatric disorder (1) diminish early perceptual processing $(<200 \mathrm{~ms})$ of emotional stimuli without substantially affecting activity modulation by stimulus arousal value, (2) diminish later attention allocation processes ( $>300 \mathrm{~ms}$ ), and (3) are related to more recent life stress. High intraindividual correlations of activity patterns between sessions suggest lasting effects of ELS on processing modes.
\end{abstract}

\section{Introduction}

Automatic (involuntary) attention capture by emotional cues is supposed to support the preparation and organization of efficient appetitive and defensive actions (Lang et al., 1998). The power of emotional stimuli to attract attention is reflected in the modulation of cortical responses by the salience and valence of affective pictures, as verified in electroencephalographic (EEG; Junghöfer et al., 2001; Schupp et al., 2004), magnetoencephalographic (MEG; Peyk et al., 2008) or functional magnetic resonance imaging (fMRI) studies (Junghöfer et al., 2006; Phan et al., 2004). Activity in posterior brain regions is augmented as early as $150 \mathrm{~ms}$ after the onset of arousing pleasant and unpleasant pictures relative to non-arousing neutral pictures. Two components of selective emotional processing have been distinguished from opposite polarity of magnetic fields and from source analyses in an earlier time interval (120-170 $\mathrm{ms}$ after stimulus onset) in occipital-parietaltemporal regions and a later time interval (220-310 ms after stimulus onset) in more anterior temporal regions (Peyk et al., 2008). Both

\footnotetext{
* Corresponding author. Department of Psychology, University of Konstanz, P.O.Box D23, D-78457 Konstanz, Germany. Tel.: +49 7531 884606; fax: +49 7531882891. E-mail address: Katharina.matz@uni-konstanz.de (K. Matz).
}

activities have been related to automatic, perceptual attention capture by salient stimuli, but seem to reflect distinct processing states in the visual stream. The modulation of early cortical activation by stimulus content seems to be robust against stimulus duration and frequency, as it has been demonstrated for stimulus duration of $1500 \mathrm{~ms}$ (Schupp et al., 2003) and rapid presentation rates between 3 and $12 \mathrm{~Hz}$ (Junghöfer et al., 2001; Peyk et al., 2009).

The cortical responses to emotional stimuli, which characterize normal subjects, are often found to differ in individuals with a psychiatric disorder. Moratti et al. (2008) found less modulation of right-hemispheric temporo-parietal activation evoked by arousing pictures in patients with major depressive disorder (MDD) than in healthy controls. Similarly, Canli et al. (2004) reported lower response amplitude to words with happy and more activity to words with sad meaning in MDD patients compared to controls. Schizophrenia patients were found to exhibit less cortical activity modulation to arousing emotional pictures than healthy subjects (Rockstroh et al., 2006), and smaller amplitudes of the P300-event-related potential evoked by negative facial expressions (An et al., 2003). Such changes are assumed to reflect characteristics of psychopathology (like flat affect, negative symptoms) rather than consequences of dampening medication (Dichter et al., 2004; Mueser et al., 1997). 
Affective processing modes may be influenced by experiences early in life. Adverse experiences early in life have been found to influence stress-sensitive systems like the hypothalamus-pituitaryadrenal (HPA) axis and cortical systems (Charmandari et al., 2005; Sterlemann et al., 2008; Champagne et al., 2008; de Kloet et al., 2005; Plotsky et al., 2005). As a consequence, psychophysiological reactivity (e.g., Pole et al., 2007; Meyer et al., 2001) may be modified including more sensitive responses to further stressors (e.g. Hazel et al., 2008; Heim et al., 2004) and altered affective processing (Lang et al., 2007; Taylor et al., 2006). Adverse experiences early in life have also been discussed as potential factor infuencing the development and course of psychiatric disorders in predisposed individuals (Andersen and Teicher, 2008, 2009; Leonardo and Hen, 2008; Walker et al., 2008; Cohen et al., 2006; Dinan, 2005; Dohrenwend, 2006; Nemeroff, 2004; Heim et al., 2004; McEwen, 2003). Heim and colleagues (Heim et al., 2003, 2004; Pace et al., 2006; Bradley et al., 2008; see also Van den Bergh et al., 2008) have demonstrated in a subtype of depression that early life stress may increase the sensitivity and reactivity of the HPAaxis, thereby affecting stress-sensitivity and stress reactivity throughout life (e.g., Graham et al., 1999). While this interaction may explain the relationship between early life stress and the course of depressive disorder later in life, it seems unclear, whether the sensitizing effect of early life stress also involves affective processing modes in the brain.

In a previous study, we examined effects of retrospectively reported adverse experiences in childhood (labeled early life stress, ELS, from here on) on cortical responses to emotional stimuli in patients with different psychiatric diagnoses (Weber et al., 2009). Adult patients who had reported a high number of stressful life events in childhood displayed reduced right-posterior activity to higharousing pleasant and unpleasant pictures 160-210 ms after stimulus onset relative to patients with low ELS and relative to non-stressed, healthy comparison subjects. The present study explored, whether similar indications of altered cortical affective processing would be evident some 1.5 years later as a sign of lasting effects of ELS. Subjects with particularly high and with low ELS were selected from the sample recruited by Weber et al. (2009), see also Weber et al., 2008) to participate in two sessions 11 and 19 months after the previous study. Processing of emotional stimuli was examined using a rapid serial visual presentation (RSVP) protocol (Junghöfer et al., 2001). If ELS exerts lasting effects on the brain's emotional processing modes, we should expect similar cortical responses to emotional stimuli across measurements and stimulation conditions. In addition, considering Heim's model of stress-sensitization by ELS mentioned above, the present study explored, whether an increased vulnerability for stressful experiences would be evident in adult subjects with high ELS and whether cortical processing of emotional stimuli might constitute a mediator between EIS and stress reactivity in adulthood. Therefore, we examined, whether subjects differing in ELS also exhibited different experiences of current life events and whether this was related to cortical responses to emotional stimuli. Specifically, the present study examined the hypotheses that (1) the previously described differences in cortical activation by emotional stimuli between individuals with and without a psychiatric disorder could be replicated, that (2) the previously described differences in cortical activation by emotional stimuli between individuals with high and low ELS were stable across time, and that (3) differences in cortical activation by emotional stimuli between individuals with high and low ELS were related to the individuals' current life stress load.

\section{Methods}

\subsection{Participants}

The present sample comprised 31 patients ( 12 females, mean age $40.0 \pm 12.6$ years) and 15 healthy subjects ( 7 females, mean age $40.7 \pm$ 16.8 years). Subjects were selected on the basis of their history of ELS assessed with the Early Trauma Inventory (ETI; Bremner et al., 2000; German version by Heim, 2000) from an initial sample of 96 psychiatric inpatients and 36 healthy subjects. The ETI determines adverse experiences in the four domains of emotional neglect, physical abuse, sexual abuse and general traumatic events for different periods of life. An ELS index was defined as the sum of products of frequency and duration for each event reported before the individual onset of puberty $^{3}$ summed up across all domains. For the present study, the 15 patients with the highest ELS scores were selected from the original sample. They were compared to 15 subjects of the healthy comparison group, who had generally displayed low stress load scores, and 16 patients with scores within the range of the comparison group. From this sample, 23 patients and 12 healthy subjects had participated in the previous MEG-study one year earlier (Weber et al., 2009).

According to ICD-10 (International Classification of Diseases, 10th Revision), patients had been diagnosed by senior psychiatrists with Major Depressive Disorder (MDD), schizophrenia, drug addiction (DA), and Borderline Personality Disorder (BPD; see Table 1 for demographic and clinical information of the present sample). Most patients were on psychoactive medication receiving combinations of antidepressant and neuroleptic, typical and atypical neuroleptic drugs, or antidepressants of tricyclic or reuptake-inhibitor type (see Table 1). At the time of the present study, the majority of patients had been released, which indicates their clinical improvement. Exceptions were long-term admissions on the forensic ward including ten patients in the first and seven in the second session, of which $3 / 1$ were drug addicts, $4 / 4$ schizophrenics and $3 / 2$ patients with BPD. As participants of the present study were not seen again by the respective hospital psychiatrists and not diagnosed again, the presently reported diagnoses refer to lifetime diagnoses.

Healthy subjects were included into the comparison group, if they had never met criteria of any psychiatric disorder according to the M.I.N.I. (Ackenheil et al., 1998) and did not take psychoactive medication. Individuals with neurological conditions, head trauma with loss of consciousness, or intellectual disability were excluded. All participants had normal or corrected to normal vision. The Edinburgh Handedness Questionnaire (Oldfield, 1970) confirmed right-handedness in 38 participants. Six participants were ambidextrous and two were lefthanded. Since analyses with and without the left-handed and ambidextrous subjects did not provide different results, analyses are reported for the entire sample.

\subsection{Design and procedure}

The study protocol was approved by the ethics committee of the University of Konstanz. All participants provided written informed consent.

The present study comprised two measurement points, which were 8 months apart. Using the Münchner Ereignisliste (MEL; MaierDiewald et al., 1983) each measurement started with the screening of life events experienced in the preceding six months. Life events were assessed in the domains of work, life, interpersonal relationships and violence. Participants were asked whether they had experienced a certain event and to rate the subjectively experienced stressfulness of this event on a 5-point-Likert scale. Thereafter, the MEG was recorded, while subjects monitored pictures in a rapid serial visual presentation (RVSP) protocol (Junghöfer et al., 2001). Based on the normative ratings of emotional valence and arousal, as well as analysis of physical picture parameters, 300 pictures from the International Affective Picture System (IAPS; Center for the Study of Emotion and Attention, 2004) were selected to three categories of 100 higharousing pleasant, 100 high-arousing unpleasant and 100 lowarousing neutral. Each stimulus was presented once within each of

\footnotetext{
${ }^{1}$ According to Heim et al. (2004), ELS accounts for the period between birth and the time of sexual maturation, the latter being determined by the onset of puberty.
} 
Table 1

Demographic and clinical characteristics of groups in the two sessions.

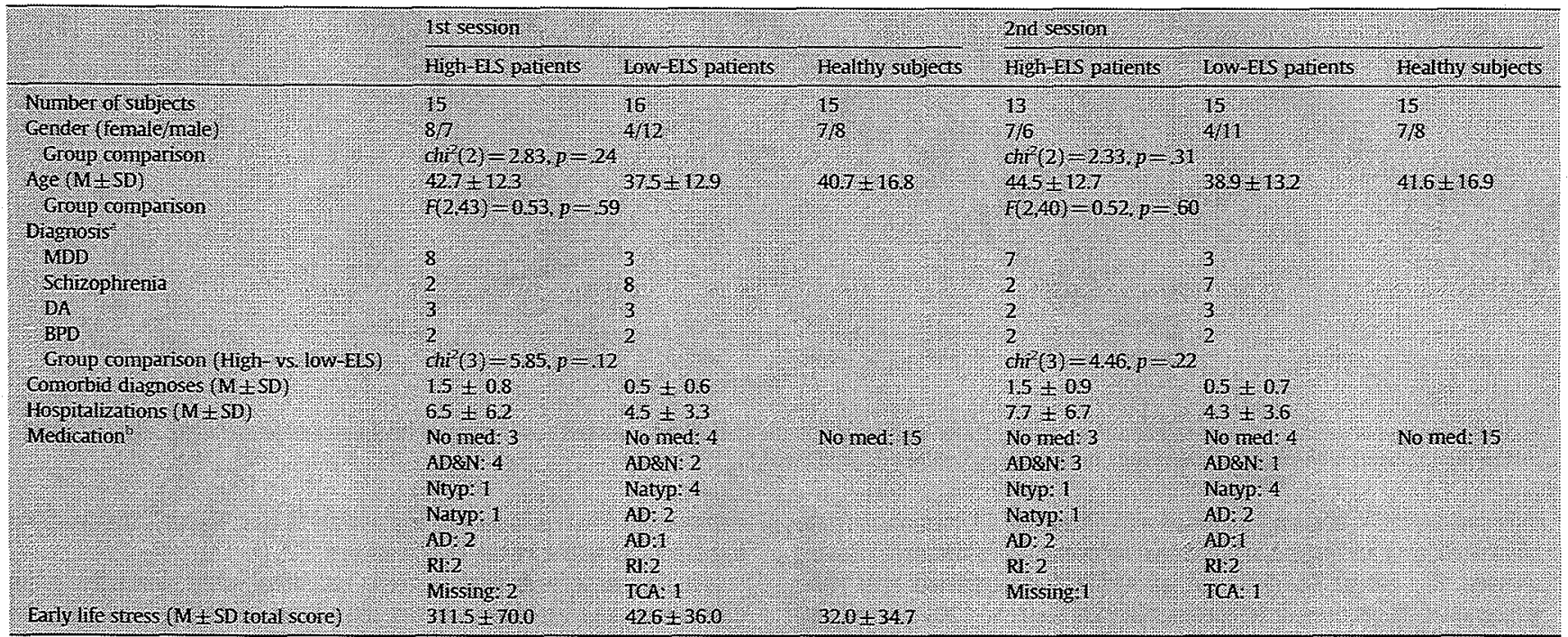

a Diagnoses: MDD: Major depressive disorder; DA: Drug addiction; BPD: Borderline personality disorder.

b Medication: AD\&N: combination of antidepressants and neuroleptics; Ntyp: typical neuroleptics; Natyp: atypical neuroleptics; AD: combination of tricyclics and serotonin/NA reuptake-inhibitors; RI: selective serotonine reuptake inhibitor or serotonine-NA-reuptake inhibitors; TCA: tricyclic antidepressives.

two series of 300 pictures (total 600 stimuli). Pictures were presented without perceivable gap for $349 \mathrm{~ms}$ each $(2.86 \mathrm{~Hz}, 60 \mathrm{~Hz}$ refresh rate) in a pseudorandom sequence. Presentation order was controlled for transition probabilities between the three stimulus categories. Physical picture parameters (brightness, contrast, color distribution, complexity) did not differ between stimulus categories. Timing and sequence of stimulus presentation were controlled using PRESENTATION software (Neurobehavioral Systems ${ }^{*}$, Albany, CA, USA). Participants were asked to keep their eyes focused on a small central fixation cross overlaying each picture and to attend to the picture series carefully without any additional task. The two picture series were presented without break. Presentation of the total 600 stimuli lasted for about $4 \mathrm{~min}$.

\subsection{Data acquisition and analysis}

The MEG was recorded while subjects were in a prone position using a 148-channel magnetometer (MAGNES'M 2500 WH, 4D Neuroimaging, San Diego, USA). Neuromagnetic data were continuously recorded with a sampling rate of $678.17 \mathrm{~Hz}$ and a bandpass filter of 0.1 to $200 \mathrm{~Hz}$. For artifact control, the vertical and horizontal electrooculogram (EOG from four electrodes placed near the left and right temporal canthus and above and below the right eye) and the electrocardiogram from two electrodes attached to the right and left forearm were recorded using a SynAmps amplifier (NEUROSCAN Laboratories, Sterling, VA, USA). The subject's nasion, left and right ear canal, and head shape were digitized with a Polhemus 3 Space ${ }^{\circledR}$ Fasttrack prior to each session.

Following noise reduction based on distant reference sensors, MEG data were corrected for heartbeat-related artifacts: In time segments with $R$-wave artifact, an average magnetocardiogram was subtracted, calculated as a moving average over 20 heartbeats (4D Neuroimaging "cardiac comber" software). Further preprocessing was accomplished with BESA ${ }^{\circledast}$ software (MEGIS Software GmbH, Munich, Germany) and included filtering of continuous data with a $0.5 \mathrm{~Hz}(6 \mathrm{~dB} /$ octave, forward-shift) high-pass and a $40 \mathrm{~Hz}$ ( $48 \mathrm{~dB} /$ octave, zero-phase-shift) low-pass filter, and rejection of epochs containing eye blinks. Data of one MDD patient from the low-ELS group had to be excluded from analyses of the first session because of too many artifact-contaminated trials.
Following preprocessing, event-related fields were averaged across trials separately for each subject and stimulus category. Of the 200 trials of each stimulus category 188 artifact-free trials were available on average for low-ELS patients, 188 trials for high-ELS patients and 197 trials for healthy subjects in the 1st session (group difference n.s.). A similar number of trials was available in the 2 nd session (185 trials for low-ELS patients, 175 trials for high-ELS patients and 198 trials for healthy subjects (difference n.s.). Each trial was referenced to the preceding trial as a baseline. Averaged across trials, baselines represented an average over the three stimulus categories. The L2-Minimum-Norm-Pseudoinverse was used for inverse modeling, providing minimum norm estimates (MNE; Hamalainen and Ilmoniemi, 1994; Hauk et al., 2002; Hauk, 2004). Relying on EMEGS ${ }^{\odot} 2.4$ custom software (Junghöfer and Peyk, 2004) written in Matlab (MathWorks Inc., MA, USA), a spherical shell with $2 \times 350$ evenly distributed dipoles (azimuthal and polar direction, radial dipoles do not generate magnetic fields outside of a sphere) served as the source model. A source shell radius of $87 \%$ of the individually fitted head radius was chosen, roughly corresponding to grey matter. A Tikhonov regularization parameter of 0.2 was applied. Independent of dipole direction, source strength was calculated as the vector length of the generator activity at each position for each subject, condition and time point based on the averaged magnetic field distributions and individual sensor positions.

The course of global power of estimated neural activity, illustrated in Fig. 1 for the 1 st session, indicates two activity peaks around $100 \mathrm{~ms}$ and 200-250 ms after stimulus onset, followed by a general decline in activity. Whereas activity differs between patients and healthy subjects around $100 \mathrm{~ms}$, differences between high-and low-ELS subjects emerge at around $200 \mathrm{~ms}$ and later. For statistical evaluation of group and stimulus effects, two sets of point-wise repeated-measures analysis of variance (ANOVA) were accomplished separately for each estimated source and time point: one ANOVA, carried out with healthy subjects only, included the within-subject factor Emotion (comparing pleasant, unpleasant and neutral stimuli). This ANOVA served to verify the modulation of cortical activation by emotional stimulus content as described, for instance, by Peyk et al. (2008, see also Schupp et al., 2006). The second ANOVA included the between-subjects factor ELS (comparing the three groups). To avoid false positives, significant effects were 


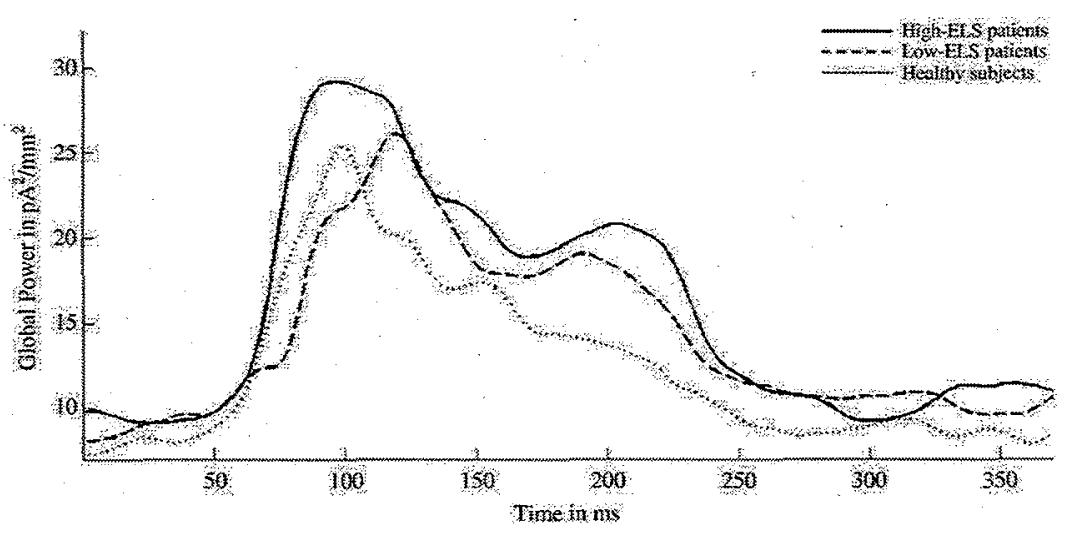

Fig. 1. Time course of overall estimated source activity (global power in $\mathrm{pA}^{2} / \mathrm{mm}^{2}$ ) in the first session, averaged across stimulus categories separately for patients with high ELS (dotted line), patients with low ElS (dashed line) and healthy comparison subjects (solid line).

only considered when they included a minimum of 21 continuous data points ( $32 \mathrm{~ms}$ ) and when at least two adjacent representative dipoles showed the effect. The first set of ANOVAs determined two time windows with prominent effects of stimulus content, $120-170 \mathrm{~ms}$ and 250-349 ms after stimulus onset. The second set of ANOVAs determined group differences for the time windows $70-120 \mathrm{~ms}$, and $170-250 \mathrm{~ms}$ after stimulus onset. In the next step, cortical regions (regions of interest, ROI), in which the differences between groups or stimulus conditions were prominent, were determined by plotting the statistical measures of activity differences (F-ratios) onto a spherical configuration of dipoles. Fig. 2 illustrates the ROIs defined as dipole groups with highly significant $F$-ratios for each time window.

Effects of psychiatric disorder and ELS on dipole activity in the four time windows were verified by two repeated-measures analyses of variance (ANOVA), one with the between-subject factor Group (comparing patients and healthy subjects), the other with the between-subject factor ELS (comparing high-ELS, low-ElS patients and healthy subjects). In both ANOVAs the effect of emotional stimulus content was evaluated by the within-subject factor Emotion (comparing pleasant, unpleasant, and neutral stimuli), and in both ANOVAs, differences of dipole activity between left and right RoI were tested with the additional within-subject factor Hemisphere. Main effects of Group or ELS should reflect overall differences in cortical responses across stimulus categories, whereas interactions between Group or ELS and Emotion should reflect group-dependent differential processing of stimulus content Post hoc analyses decomposed significant main effects or interactions with orthogonal polynominal contrasts and follow-up pair-wise comparisons corrected with Bonferroni, with polynominal contrasts capturing the effect of stimulus valence (pleasant vs. unpleasant) as a linear trend and the effect of stimulus arousal (pleasant and unpleasant vs. neutral) as a quadratic trend. These trends reflect a priori hypotheses about critical dimensions of emotion (e.g. Lang et al., 1998). Effects of ELS (ELS-score as a measure of severity and age at the first reported event as a measure of ELS-onset) on dipole activity were probed by intraindividual partial correlations $(r)$ using the number of reported life events as a control variable. In addition, effects of disorderseverity on dipole activity were probed by non-parametric spearman correlations with the number of hospitalizations and the number of comorbid diagnoses. In order to control for potential gender effects on cortical activity (Sabatinelli et al., 2004), an additional ANOVA with the between-subject factor Gender and the within-subjects factors Emotion and Hemisphere was accomplished. A main effect Gender only emerged in the $120-170 \mathrm{~ms}$ interval in the 1 st session $(F(1,43)=8.12, p<.01)$ with men displaying stronger cortical responses than women irrespective of stimulus valence. Since there were no interactions with Emotion or Hemisphere in any of the four components, results are reported for men and women together. Temporal stability of cortical activity across sessions was explored using Pearson correlations $(r)$ and an additional exploratory ANOVA including the within-subject factor Time (comparing dipole activities of the two sessions).

Finally, as interview data were not distributed normally. group differences and variations across the two sessions regarding current life stress were evaluated with non-parametric tests (Kruskal-Wallis $\left(c h i^{2}\right)$ and Mann-Whitney-U $(U)$ for independent, Wilcoxon signedrank test $(T)$ for dependent measures). The relationship between current life stress and cortical responses was probed by nonparametric Spearman correlations.

For all analyses statistical significance was evaluated at the .05 level. Possible violations of the homogeneity of covariance assumption were corrected with the Huynh-Feldt epsilon (statistical reports include uncorrected degrees of freedom and epsilon-corrected $p$-values).

\section{Results}

\subsection{Processing of emotional stimuli is modified by psychiatric disorder}

Lower dipole activity in patients as opposed to healthy subjects, as illustrated in Fig. 1 for the 1st session, was statistically confirmed for the $70-120 \mathrm{~ms}$ and the $170-250 \mathrm{~ms}$ interval (main effect Group, $p<.05$; see also Table 2). In the 2 nd session, group differences were verified for all three latency windows between 70 and $250 \mathrm{~ms}$.

In patients and controls, modulation of occipital-parietal dipole activity by high-arousing pleasant and unpleasant relative to lowarousing neutral stimuli was evident $120-170 \mathrm{~ms}$ following stimulus onset (Emotion, 1st session, $F(2,86)=10.94, p<.0001, \varepsilon=1.0$; linear trend explaining $19 \%$ of the variance, $F(1,43)=4.16, p<.05$; quadratic trend explaining $81 \%$ of the variance, $F(1,43)=17.97, p<.001)$. In the 2nd session, the Emotion effect $(F(2,82)=4.62, p<.05, \varepsilon=1.0)$ was carried by a linear trend indicating the largest responses to unpleasant stimuli (linear trend explaining $85 \%$ of the variance, $F(1,41)=8.90$, $p<.01$, quadratic trend explaining $15 \%, F(1,41)=1.15, p>.2$ ). At $250-$ $349 \mathrm{~ms}$, groups differed in the modulation of dipole activity by emotional content (Group $\times$ Emotion, 1st session, $F(2,86)=5.97$, $p<.01$, 2nd session, $F(2,82)=6.52, p<.01)$ : healthy subjects exhibited most pronounced parietal-temporal dipole activity in response to pleasant stimuli (linear trend explaining $72 \%$ of the variance in the 1st session, $F(1,14)=18.67, p<.001$, and $67 \%$ in the 2 nd session, $F(1,14)=$ $10.41, p<.01$ ), whereas modulation by arousal prevailed in patients (1st session, quadratic trend explaining $60 \%$ of the variance, $F(1,29)=4.95$, $p<.05 ; 2$ nd session, $97 \%, F(1,29)=3.90, p=.06$ ).

\subsection{Processing of emotional stimuli is modified by ELS}

If ELS was related to lasting changes in cortical affective processing, we might expect a relationship between ELS and present estimated neural activity. Across all subjects, correlation coefficients 

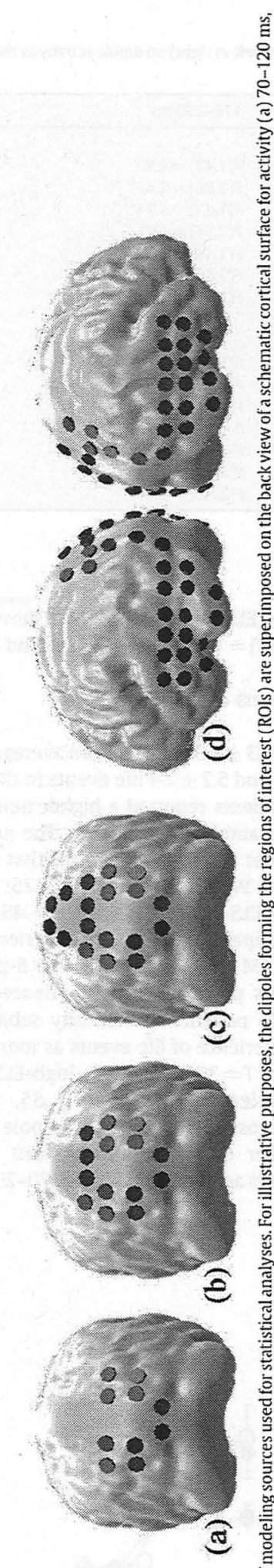

indicated that higher ELS was moderately related to lower overall dipole activity (all components, all stimulus categories, both hemispheres; 1 st session: $r=-.31, p<.05 ; 2$ nd session: $r=-.36, p<.05$ ).

Figs. 1 and 3 indicate lower estimated source activity in high-ELS patients compared to low-ELS patients and healthy subjects around $200 \mathrm{~ms}$ and thereafter. This was confirmed in the repeated measure ANOVA for the $170-250 \mathrm{~ms}$ component (ELS 1st session: $F(2,42)=$ $4.29, p<.05$; 2nd session: $F(2,40)=5.70, p<.01$ ). Bonferroni post hoc tests verified significant differences between high-ELS and healthy subjects ( 1 st session, $p<.05$, 2nd session, $p<.01$ ), whereas low-ELS patients did not differ significantly from the other two groups. Correlations between higher ELS scores and lower neural activity across subjects were also confirmed for this time window (1st session: $r=-.40$, 2nd session: $r=-.40, p<.01$ ). ELS-onset (measured by the age at the first reported event) and dipole activity at $170-250 \mathrm{~ms}$ were significantly correlated across subjects in the first $(r-.34, p<.05)$ but not in the second session. Neither within the patient group nor within the diagnostic subgroups, relationships between dipole activity and severity of illness (measured by the number of hospitalizations and the number of comorbid diagnoses) were significant.

All subjects showed modulation by arousal between $120-170 \mathrm{~ms}$, which persisted in high-ELS patients in the subsequent $170-250 \mathrm{~ms}$ window (1st session, ELS $\times$ Emotion, $(F(4,84)=2.44, p=.05$; main effect Emotion for high-ELS patients $F(2,28)=4.92, p<.05$, quadratic trend explaining $52 \%$ of the variance, $F(1,14)=6.39, p<.05)$. In the later $250-349 \mathrm{~ms}$ time interval an interaction ELS $\times$ Emotion (1st session, $F(2,84)=3.02, p<.05$; 2 nd session: $F(4,80)=3.20, p<.05$; main effects Emotion, 1st session, $F(2,84)=15.22, p<$. 001; 2nd session, $F(2,80)=8.14, p<.001$ ) resulted from pronounced activation by pleasant stimuli in healthy subjects (linear trend, $F(1,14)=18.67$, $p<.001)$ compared to low-ELS patients, who tended to show a dominant modulation by arousal (quadratic trend, $F(1,14)=2.97$, $p=.1$ ), and high-ELS patients, who exhibited neither arousal nor valence modulation (quadratic trend, $F(1,14)=1.91, p=.19$; linear trend, $F<1$ ). Stronger activation by pleasant stimuli in this time interval was related to later ELS-onset across subjects in both sessions (1st session: $r=.37,2$ nd session: $r=.34, p<.05$ ), but there were no significant correlations with ELS-severity. Neither within the patient group nor within the diagnostic subgroups, relationships between cortical responses to pleasant stimuli and severity of illness were significant.

Right-hemispheric dominance of dipole activity was verified for all subjects around $200 \mathrm{~ms}(120-170 \mathrm{~ms}$ or $170-250 \mathrm{~ms}$, see Tables 2 and 3 for Hemisphere effects). For the $170-250 \mathrm{~ms}$ component, an Emotion $\times$ Hemisphere interaction (1st session, $F(2,86)=4.66$, $p<.05, \varepsilon=.93 ; 2$ nd session, $120-170 \mathrm{~ms}, F(2,82)=4.09, p<.05$; $170-250 \mathrm{~ms}, F(2,82)=3.60, p<.05)$ confirmed that the described modulation of estimated neural activity by emotional stimulus content was confined to the right hemisphere.

\subsection{Stability of ELS effects}

Fig. 4 illustrates high intraindividual correlation, that is, stability of cortical responses to emotional stimuli across the two measurements. Pearson correlation coefficients were significant for the four components and the three stimulus categories ( $70-120 \mathrm{~ms}: r=.70$ to $r=.86$; $120-170 \mathrm{~ms}: r=.67$ to $r=.81 ; 170-250 \mathrm{~ms}: r=.76$ to $r=.85 ; 250-$ $349 \mathrm{~ms}: r=.36(p<.05)$ to $r=.73$, all $p<.01)$, indicating similarity of response patterns across time. The ANOVA including the withinsubjects factor Time disclosed larger early ( $70-120 \mathrm{~ms})$ dipole activity in the 2 nd compared to the 1 st session in all subjects $(F(1,40)=9.23$, $p<.01, \varepsilon=1.0)$. In healthy subjects, larger activity in the 2 nd session was also found at $170-250 \mathrm{~ms}$ (Time $\times$ Group, $F(1,40)=4.26, p<.05$; Time, $F(1,40)=10.82, p<.01 ; \varepsilon=1.0$ ). Stability of ELS effects across time can be taken also from similarity of effects across the two sessions, as summarized in Tables 2 and 3. Exceptions from similarity have to be 
Table 2

Statistical effects of Group (patient vs comparison), Emotion (pleasant vs unpleasant vs neutral stimuli) and Hemisphere (left vs right) on dipole activity in the four latency windows, separately for the two sessions.

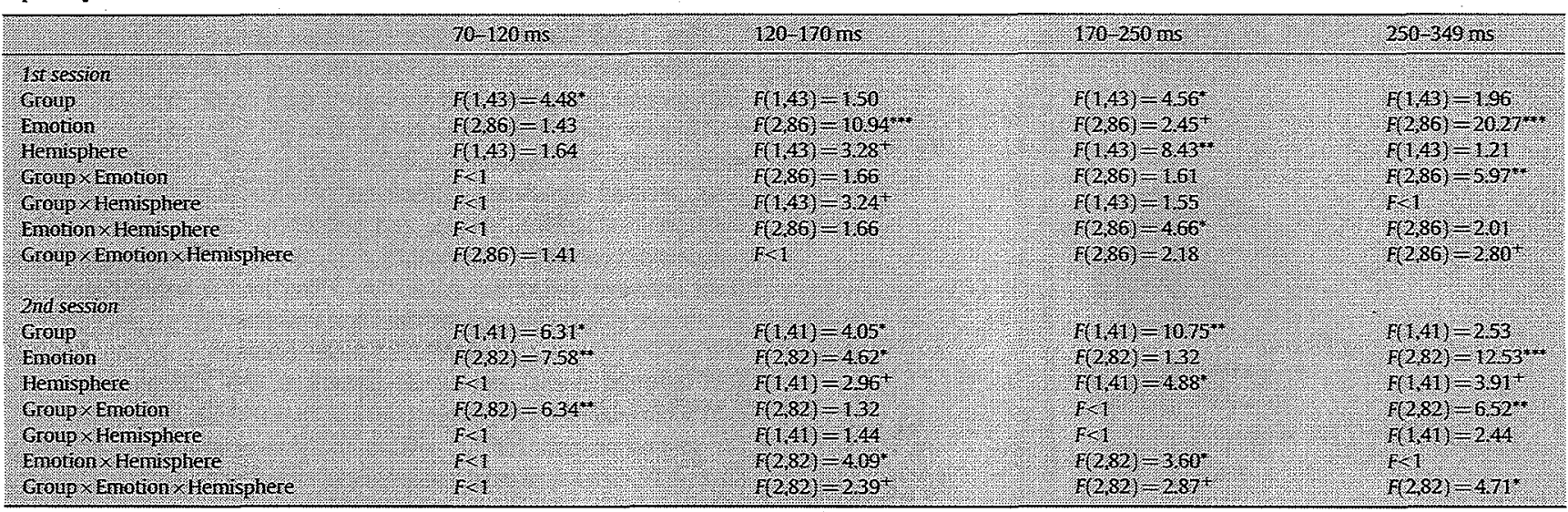

${ }^{*}: p<.05,{ }^{* *}: p<.01 ;{ }^{* * *}: p<.001 ;+p<.1, F$-ratios without superscript: $p>.1$.

noted: (1) in the 2 nd session dampened dipole activity and groupspecific emotional modulation of dipole activity in high-ELS patients became prominent already in the earliest time window $70-120 \mathrm{~ms}$ after stimulus onset, which was not the case in the 1st session (see Tabie 3 , 2nd session: main effect ELS, $F(2,40)=3.14, p<.05$, ELS $\times$ Emotion, $F$ $(4,80)=3.14, p<.05$. Emotion, $F(2,80)=5.0, p<.01)$. The arousal modulation was most pronounced in high-ELS patients (quadratic trend $(F(1,12)=5.27, p<.05)$. Low-ELS patients did not show any modulation by stimulus content (quadratic trend $F(1,14)=1.78$, $p=.20)$. The interaction Group $\times$ Emotion in Table $2(F(2,82)=6.34$, $p<.05$; Emotion, $F(2,82)=7.58, p<.01, \varepsilon=0.97$ ) may be explained as a related effect. (2) In contrast-or as a consequence-emotional modulation at $170-250 \mathrm{~ms}$ was weaker in the 2 nd compared to the 1st session, so that interactions of Emotion with ELS or Hemisphere were no longer significant (see Table 3). Since the Emotion $\times$ Hemisphere effect, which was significant at $170-250 \mathrm{~ms}$ in the 1 st session, became significant for the earlier $120-170 \mathrm{~ms}$ window in the 2nd session (see Table 3 ), one may again speculate that modulation started slightly earlier in the 2nd compared to the 1st session. (3) Emotional modulation in the two patient groups (high- and low-ELS) at 250$349 \mathrm{~ms}$ were reverse in the 2 nd relative to the 1st session: high-ELS patients displayed an arousal effect (quadratic trend $F(1,12)=5.07$, $p<.05$ ), whereas low-ELS patients did not show any modulation (quadratic trend $F(1,14)=1.13, p=.31$ ) in the 2 nd session.

\subsection{ELS, current life stress and cortical activity}

Subjects reported $5.3 \pm 2.9$ life events on average for the preceding six months in the 1st and $5.2 \pm 2.4$ life events in the 2nd session (see Table 4). High-ELS patients reported a higher number of life events than low-ELS patients and healthy subjects. The number of reported life events did not differ across the 8 months that separated the two sessions (total sample: Wilcoxon $T=447, p=.75$; high-ELS patients, $T=45.5, p=1.0$; low-EIS patients, $T=47, p=.46$; healthy subjects, $T=52, p=65$ ). Participants subjectively experienced life events as only mildly stressful ( $M \pm S D 2.2 \pm 1.0$ on the 5-point-Likert scale). Still, high-ELS patients perceived the experienced events as more stressful than low-ELS patients and healthy subjects. Within eight month, subjective experience of life events as more stressful did not change (total sample: $T=396.5, p=.66$; high-ELS patients: $T=25$, $p=.27$, low-ELS patients: $T=49.5, p=.85$, healthy subjects $T=37.5, p=.20$ ). Across subjects, lower dipole activity was not related to the number of stressful events but to the subjective experience of life events as more stressful at $170-250 \mathrm{~ms}$ (1st session,

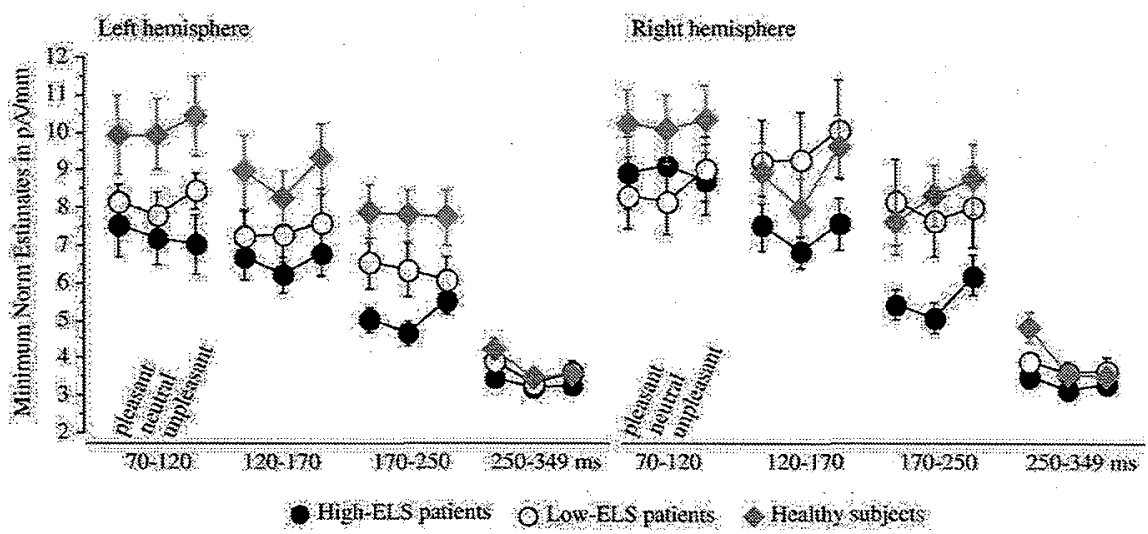

Fig. 3. Estimated source activities in the 1st session are plotted for four latency windows (abscissa: $70-120 \mathrm{~ms}, 120-170 \mathrm{~ms}, 170-250 \mathrm{~ms}$, 250-349 ms after stimulus onset) separately for emotional stimulus content (pleasant-neutral-unpleasant), group (filled circles: high-ELS patients, open circles: low-ELS patients, grey squares: healthy subjects) and left and right hemisphere. Ordinate: Dipole activity expressed as minimum norm estimates, Mean \pm Standard Error in pA/mm. 
Table 3

Statistical effects of ElS (high-ELS vs low-ELS patients vs comparison), Emotion (pleasant vs unpleasant vs neutral stimuli) and Hemisphere (left vs right) on dipole activity in the four latency windows, separately for the two sessions.

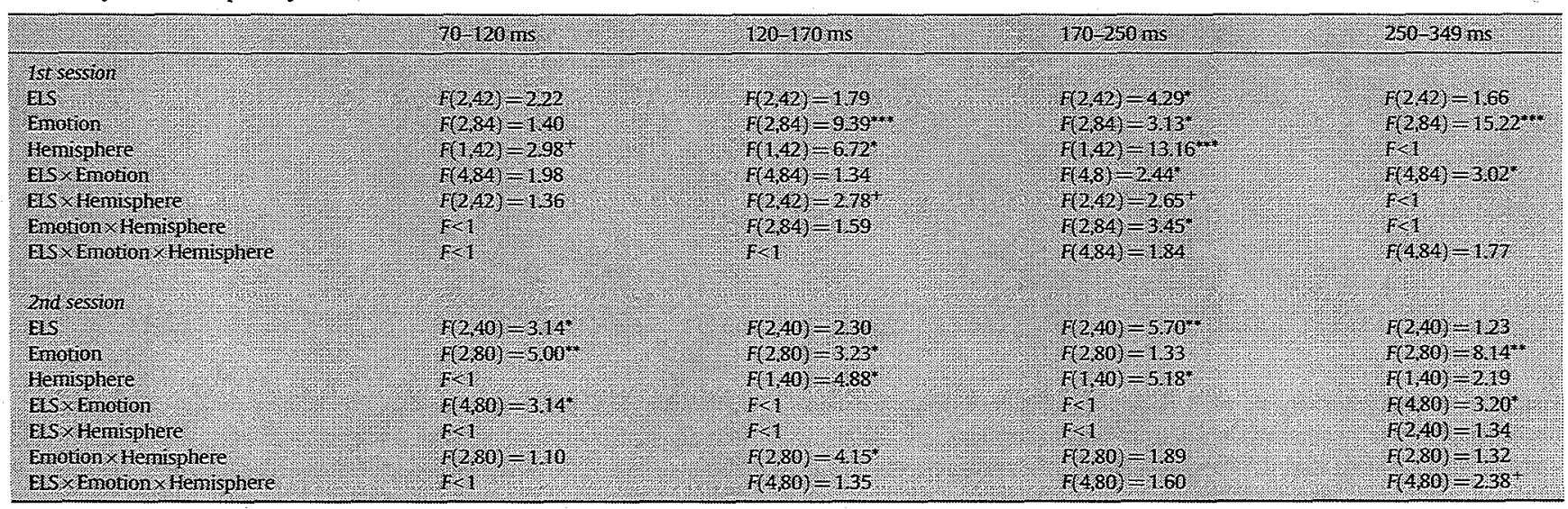

: $p<.05 .{ }^{* *}: p<.01 ;{ }^{* * *}: p<.001{ }^{+}: p<.1$, F-ratios without superscript; $p>.1$.

$r_{s}=-.37,2$ nd session, $\left.r_{s}=-.28, p=.07\right)$ and at $250-349 \mathrm{~ms}(1 \mathrm{st}$ session, $\left.r_{s}=-.36, p<.05\right)$.

\section{Discussion}

The present study explored long-term effects of early life stress on cortical affective processing modes, the stability of these effects across an eight-month interval, and their relation to current life stress in psychiatric patients. Lifelong effects of early life experiences on physiological and psychological functions have been verified in animal studies (Plotsky et al., 2005; Spinelli et al., 2009). In humans, they have been inferred from the relationship between early life stress, abnormal HPA-axis functioning, and severity of disorder in a subgroup of depressive patients (Heim et al., 2003, 2008; Bradley et al. 2008). Longitudinal or prospective studies in humans (e.g.. Landrigan et al.. 2008) disclosed an effect of additional stressful experiences later in development on the relationship between childhood stress and adult depression (e.g. Hazel et al., 2008) and effects of early abuse on lasting psychological distress (Lindhorst et al., 2009).

Comparing subgroups of patients with high and low ELS, present results confirmed a relationship between early life stress and abnormal cortical activity to affective pictorial stimuli. As reported for a slightly different stimulation design (Weber et al., 2009), higher ELS varied with dampened cortical responses to emotional stimuli. Lasting effects of childhood experiences were further suggested by the temporal stability of altered cortical affective processing over about eight months. Temporal stability of cortical responses to affective visual stimuli has been documented across a period of ten days (Codispoti et al., 2007). We are not aware of other studies examining stability of cortical responses and their relationship to events earlier in life across a similar period of time. The present results suggest the challenging hypothesis that the stability of ELS effects on cortical processing modes may be considered parallel to the lifelong effects reported in animal studies. This needs to be substantiated in further studies.

Dampened cortical responses to emotional stimuli seem intriguing, as one might expect more pronounced responses to emotionally arousing stimuli as a consequence of a sensitizing effect of ELS. Stresssensitization as a result of ELS has been demonstrated for endocrinological responses (e.g. Charmandari et al., 2003; Danese et al.; 2008, Heim et al., 2008) and sensitivity to further life stress (e.g. Espejo et al., 2007; Wright et al., 2009; Matz et al., in press). In patients suffering from PTSD, early enhanced frontal activation in response to emotional stimuli has been found in MEG studies (Junghöfer et al., 2003; Borgelt et al., 2009). Functional MRI studies found augmented subcortical activity in the amygdala and insula (e.g. Etkin and Wager, 2007; Morey et al., 2008). On the other hand, PTSD patients also exhibited reduced activation in other regions associated with the experience and regulation of emotion like anterior cingulate and prefrontal structures (Etkin and Wager, 2007). However, these patients experienced traumatic stress in late adolescence or

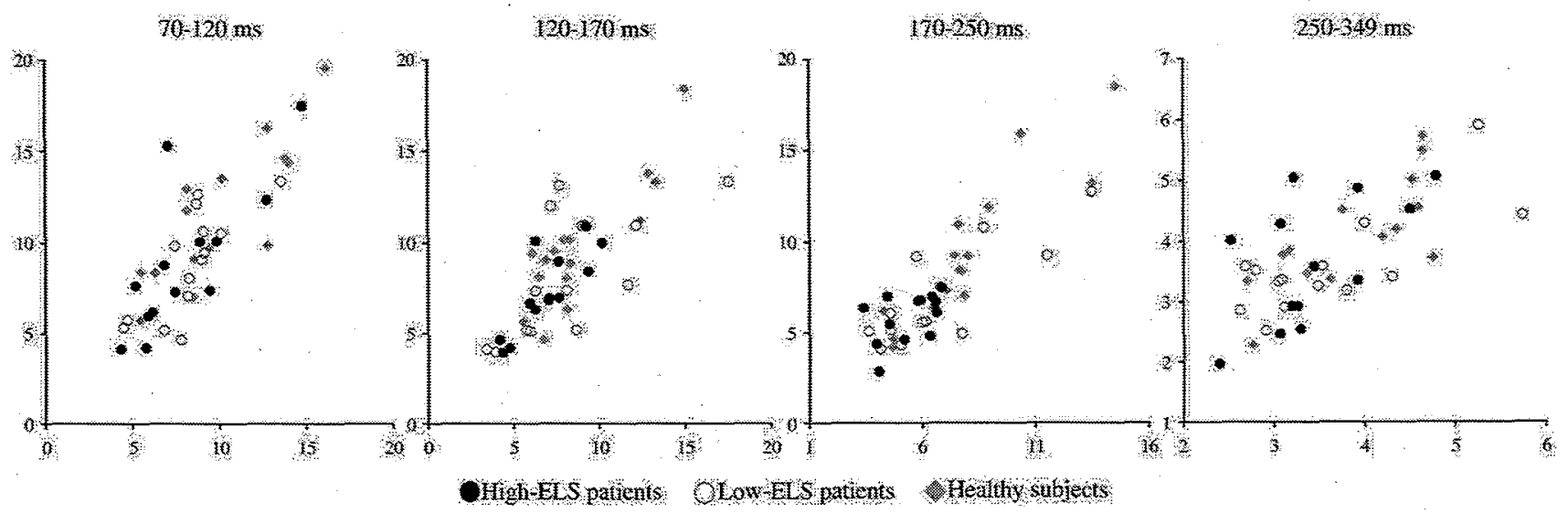

Fig. 4. Mean estimated source activity in pA/mm (across stimulus categories and hemisphere) in the 1st session (ordinate) is plotted against mean activity in the $2 \mathrm{nd}$ session (abscissa) for each subject separately for the four components (filled circles: high-ElS patients, open circles: low-ElS patients, grey squares: healthy subjects). 
Table 4

Life events and their experienced strain during the six month preceding the 1st and 2nd sessions.

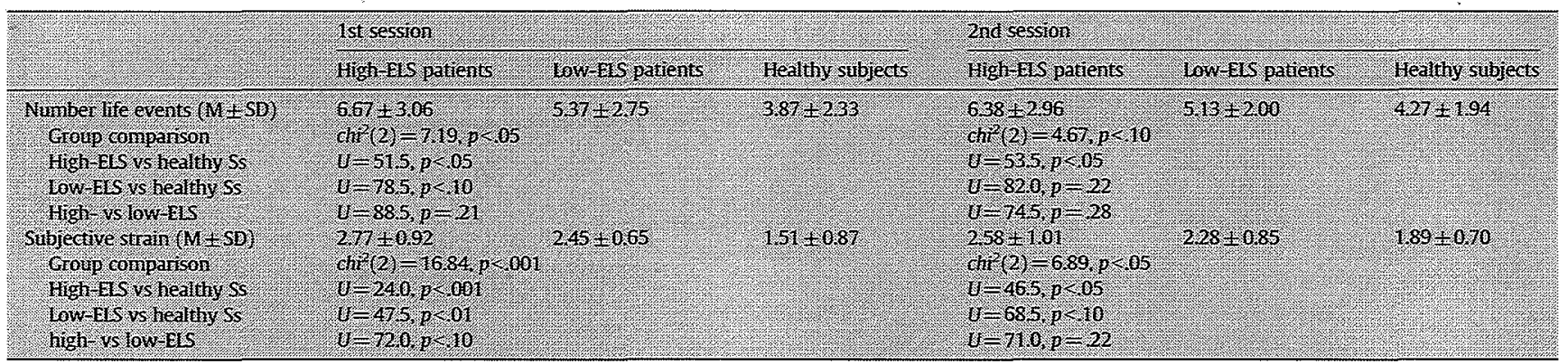

adulthood and intensity as well as timing of stress may modulate cortical affective processing in different ways (Lupien et al., 2009). It seems also possible that dampened cortical activation reflects stressrelated functional brain changes, as Tomoda et al. (2009) found reduced grey matter volume in primary and secondary visual cortices in subjects with a history of childhood sexual abuse. Moreover, white matter tract abnormalities indicating alterations in neural pathways have been associated with parental verbal abuse (Choi et al., 2009). While cortical activity can be reliably determined with the localization methods used in the present study, it is difficult to draw conclusions on (possibly enhanced) subcortical activation and on alterations in neuroanatomical pathways that might be related to dampened activation in the visual cortex. Finally, dampened cortical responses to emotional stimuli (Moratti et al., 2008) and delayed and reduced P300 responses to target stimuli (Kemp et al., 2009) have been reported for depressive patients. These results have been interpreted as an impairment of attention allocation. As depressive patients with high ELS constituted a substantial subgroup of the present sample, diagnosis-specific or -related clinical aspects might have resulted in the dampened response to pleasant stimuli around $300 \mathrm{~ms}$. Although available clinical variables (number of hospitalizations and number of comorbid diagnoses, which may roughly point to severity and duration of illness) were not related to the reported cortical activity, we cannot rule out the impact of disorder-specific aspects. The present sample may also reflect the effects of ELS on vulnerability for and course of MDD in a subgroup of (genetically) predisposed individuals, as modeled by Heim et al. (2008). Specification of the distinct effects of the two factors, depression and ELS, requires larger and more balanced samples than available for the present study. However, in the light of assumptions of gene environment interactions, isolated main effects of either ELS or diagnosis seem unlikely (e.g., Kendler et al., 2005; Turkheimer, 2000; Bradley et al., 2008).

The hypotheses, that ELS influences vulnerability for and reactivity to current stressors in adulthood, and that emotional processing might contribute to this lasting stress-sensitivity was partly supported: ELS predicted the perception of current life events as more stressful in the present adult sample. Cortical responses to emotional stimuli were related to ELS, but less prominent to current stresssensitivity. Thus, although present results may be considered a preliminary indication that affective cortical processing modes may constitute a mediating factor between ELS and psychological dysfunctions in adulthood, the mediating pathway remains to be specified in future studies for larger samples. Moreover, it should be emphasized that the reported correlations do not bear causal relationships.

The present design addressed specific aspects of cortical affective processing modes. Posterior activity in the latency range $150-300 \mathrm{~ms}$ after stimulus onset has been related to automatic perceptual processing and attention capture by emotional stimuli (Junghöfer et al., 2006; Lang et al., 1998; Schupp et al., 2003, 2004, 2006; Codispoti et al., 20 07; LeDoux, 2000). Despite of an overall reduced response, ELS did not substantially influence the modulation of activity by the arousal and valence of stimuli around $200 \mathrm{~ms}$. Around $300 \mathrm{~ms}$, high-ELS patients did not show the 'normal' response to pleasant stimuli in the first assessment, whereas arousal modulation was found in the second assessment. This suggests reduced modulation by stimulus valence. Taken together, ELS differentially affected activity in early and late time intervals that are assumed to reflect distinct states in visual emotional processing (Peyk et al., 2008). Results suggest a dampened though still sufficiently functioning automatic attention capture by emotional stimuli.

The small and unbalanced samples of the present study are certainly a major methodological shortcoming and limit the conclusions. Longitudinal studies bear the problem of drop outs and the repeated study of selected samples even amplified this problem, resulting in subsamples too small to adequately evaluate the interaction of ELS and disorder-specific influences on brain activation. Moreover, modulation of cortical responses by emotional arousal of stimulus content in healthy subjects of the present study was confined to an earlier and smaller latency window (120-170 ms) compared to results reported in the literature for normal subjects (e.g. Peyk et al., 2008, 2009). The present healthy sample differed from those 'normal' subject groups with respect to age, as subjects were recruited to be comparable to the patient samples. Moreover, the small sample size may have increased variability, and thereby reduced significant effects.

Despite of these limitations, the present results point to lasting effects of adverse childhood experiences on cortical processing modes and stress-sensitivity. Further validation of a potential specific impairment of evaluation of the intrinsic significance of emotional stimuli should improve our understanding of the relationship between adverse experiences early in life and the course of mental illness.

\section{Acknowledgements}

Research was supported by the Deutsche Forschungsgemeinschaft (FOR751). We are grateful to B. Awiszus, A. Steffen, C. Pietrek, K. Helmbold for assistance in MEG recording, interviewing and data preprocessing, and to Prof. Dr. H. Schupp for advice in data analyses.

\section{References}

Ackenheil, M., Stotz-Ingenlath, G., Dietz-Bauer, R., Vossen, A., 1998. M.I.N.l. Mini International Neuropsychiatric Interview; German Version. University of Munich, Department of Psychiatry, Germany.

An, S.K., Lee, S.]., Lee, C.H., Cho, H.S., Lee, P.G., Lee, C., Lee, E., Roh, K., Namkoong, K., 2003. Reduced $\mathrm{P} 3$ amplitudes by negative facial emotional photographs in schizophrenia. Schizoph. Res. 64, 125-135.

Andersen, S.L, Teicher, M.H., 2008. Stress, sensitive periods and maturational events in adolescent depression. Trends Neurosc. 31, 183-191.

Andersen, S.L. Teicher. M.H., 2009. Desperately driven and no brakes: developmental stress exposure and subsequent risk for substance abuse. Neurosci. Biobehav. Rev. 33, 516-524.

Borgelt, J., Odenwald, M., Ruf, M., Elbert, T., Kissler, J., submitted for publication. Rapid orbito-frontal responses to aversive stimuli in Post Traumatic Stress Disorder revealed by Magnetoencephalography. 
Bradley, RG., Binder, E.B., Epstein, M.P., Tang, X. Nair, H.P., Liu, W., Gillespie, CF, Berg, T., Evces, M, Newport, J., Stowe, ZN., Heim, CM. Nemeroff, C.B, Schwart, A., Cubells, J.F, Ressler, K J. 2008. Influence of child abuse on adult depression. Arch. Gen. Psychiatr. 65, 190-200.

Bremner, J.D., Vermetten, E., Mazure, C.M., 2000. Development and preliminary psychometric properties of an instrument for the measurement of childhood trauma: the Early Trauma Inventory. Depress. Anxiety 12, 1-12

Canli, T. Sivers, H., Thomason, M.E. Whittield-Gabrieli, S. Gabrieli, J.D., Gotlib, I.H., 2004. Brain activation to emotional words in depressed vshealthy subjects. NeuroReport 15. 2585-2588.

Center for the Study of Emotion and Attention, 2004. The International affective picture system: digitized photographs. University of Florida, Center for Research in Psychophysiology, Gainesville.

Champagne, D.L., Bagot, R.C., van Hasselt, F., Ramakers, G., Meaney, M.J., de Kloet, E.R., Joels, M, Krugers, $H_{\text {, }}$ 2008. Matemal care and hippocampal plasticity: evidence for experience-dependent structural plasticity, altered synaptic functioning, and differential responsiveness to glucocorticoids and stress. J. Neurosci. 28, 6037-6045.

Charmandari, E., Kino, T., Souvatzoglou, E., Chrousos, G.P., 2003. Pediatric stress: hormonal mediators and human development. Horm. Res. 59, 161-179.

Charmandari, E., Tsigos, C., Chrousos. G., 2005. Endocrinology of the stress response. Annu. Rev. Physiol. 67, 259-284.

Choi, J. Jeong, B., Rohan, M.L., Polcari, A.M., Teicher, M.H., 2009. Preliminary evidence for white matter tract abnormalities in young adults exposed to parental verbal abuse. Biol. Psychiatr. 65, 227-234.

Codispoti, M., Ferrari, V., Bradiey, M.M. 2007. Repetition and event-related potentials: distinguishing early and late processes in affective picture perception. I. Cognit Neurosci. 19, 577-586.

Cohen, RA., Grieve, S., Hoth, K.F., Paul, R.H., Sweet, L, Tate, D., Gunstad, J., Stroud, L, McCaffery, J., Hitsman, B., Niaura, R, Clark, C.R, MacFarlane, A, Bryant, R., Gordon, E., Williams, LM. 2006. Early life stress and morphometry of the adult anterior cingulate cortex and caudate nuclei. Biol. Psychiatr. 59, 975-982.

Danese, A., Moffitt, T.E., Pariante, C.M., Ambler, A., Poulton, R, Caspi, A, 2008. Elevated inflammation levels in depressed adults with a history of childhood maltreatment. Arch. Gen. Psychiatr. 65, 409-415.

de Kloet, ER, Sibug, RM., Helmerhorst, FM. Schmidt M.V., 2005. Stress, genes and the mechanism of programming the brain for later life. Neurosci. Biobehav. Rev. 29, 271-281.

Dichter, G.S., Tomarken, AJ, Shelton, R.C. Sutton, S.K. 2004. Early- and late-onset startie modulation in unipolar depression. Psychophysiology 41, 433-440.

Dinan, T.G., 2005. Stress: the shared common component in major mental illnesses. Eur. Psychiatr. 20 (Suppl 3), S326-S328.

Dohrenwend, B.P. 2006. Inventorying stressful life events as risk factors for psychopathology: toward resolution of the problem of intracategory variability. Psychol. Bull. 132, 477-495.

Espejo, E.P., Hammen, C.L, Connolly, N.P., Brennan, PA., Najman, J.M., Bor, W., 2007. Stress sensitization and adolescent depressive severity as a function of childhood adversity: a link to anxiety disorders. J. Abnorm. Child Psych. 35, 287-299.

Etkin, A. Wager, T.D., 2007. Functional neuroimaging of anxiety: a meta-analysis of emotional processing in PTSD, social anxiety disorder, and specific phobia. Am. J. Psychiatr. 164, 1476-1488.

Graham, Y.P., Heim, C.M., Goodman, S.H., Miller, A.H., Nemeroff, C.B., 1999. The effects of neonatal stress on brain development: implications for psychopathology. Dev. Psychopathol. 11, 545-565.

Hamalainen, M.S., Ilmoniemi, R.J., 1994. Interpreting magnetic helds of the brain: minimum norm estimates. Med. Biol. Eng. Comput 32, 35-42.

Hauk, 0., 2004. Keep it simple: a case for using classical minimum norm estimation in the analysis of EEG and MEG data. Neuroimage 21, 1612-1621.

Hauk, O. Keil, A, Elbert, T. Muller, M.M. 2002 Comparison of data transformation procedures to enhance topographical accuracy in time-series analysis of the human EEG. J. Neurosci. Meth. 113, 111-122.

Hazel, N.A, Hammen, C, Brennan, P.A., Najman, J., 2008. Early childhood adversity and adolescent depression: the mediating role of continued stress. Psychol. Med. 38, 581-589.

Heim, C., 2000. Deutsche Version des Early Trauma Inventory: Inventar zur Erfassung früher traumatischer Lebensereignisse (IFTL). Unpublished manuscript, Emory University School of Medicine, Department of Psychiatry and Behavioral Sciences, Atlanta.

Heim, $C$. Meinlschmidt, $G_{\text {.. }}$ Nemeroff, $C$, 2003. Neurobiology of early life stress. Psychiatr. Ann. 33, 18-26.

Heim, C., Plotsky. P.M., Nemeroff, C.B., 2004. Importance of studying the contributions of early adverse experience to neurobiological findings in depression. Neuropsychopharmacology 29, 64i-648.

Heim, C.M., Newport, D.J., Mletzko, T., Miller, A.H., Nemeroff, C.B., 2008. The link between childhood trauma and depression: insights from HPA axis studies in humans. Psychoneuroendocrinology 33, 693-710.

Junghöfer, M., Peyk, P., 2004. Analysis and visualization of measurements of electrical potentials and electromagnetic fields. MATLAB select 2, 24-28.

Junghöfer, M., Bradley, M.M., Elbert, T.R., Lang. P.J., 2001. Fleeting images: a new look at early emotion discrimination. Psychophysiology 38, 175-178.

Junghōfer, M., Schauer, M., Neuner, F., Odenwald, M., Rockstroh, B., Elbert, T., 2003. Enhanced fear-network in torture survivors activated by RSVP of aversive material can be monitored by magnetic source imaging. Psychophysiology 40 (Suppl.3).

Junghöfer, M., Sabatineili, D., Bradley, M.M., Schupp, H.T., Elbert, T.R., Lang, P.J., 2006. Fleeting images: rapid affect discrimination in the visual cortex. NeuroReport 17 225-229.

Kemp, A.H., Pe Benito, L, Quintana, D.S., Clark, C.R, McFarlane, A. Mayur, P., Harris, A. Boyce, P., Williams, LM., 2009. Impact of depression heterogeneity on attention: an auditory oddball event related potential study. J. Affect. Disord. doi:10.1016/.j.jad. 2009.08.010.
Kendler, K.S., Kuhn, J.W., Vittum, J., Prescott, C.A., Riley, B., 2005. The interaction of stressful life events and a serotonin transporter polymorphism in the prediction of episodes of major depression: a replication. Arch. Gen. Psychiatr. 62, 529-535.

Landrigan, P.]. Forman, J., Galvez, M., Newman, B., Engel, S.M., Chemtob, C, 2008. Impact of September 11 world trade center disaster on children and pregnant women. Mt. Sinai J. Med. 75, 129-134.

Lang, P.J., Bradley, M.M., Cuthbert, B.N., 1998. Emotion, motivation, and anxiety: brain mechanisms and psychophysiology. Biol. Psychiatr. 44, 1248-1263.

Lang, P.J., McTeague, L, Cuthbert, B.N., 2007. Fear, anxiety, depression, and the anxiety disorder spectrum: a psychophysiological analysis. In: Baker. T., Bootzin, R. Treat, T. (Eds.), Psychological Clinical Science: Recent Advances in Theory and Practice. Erlbaum, Hillsdale, N.J., pp. 167-195.

LeDoux, J.E., 2000. Emotion circuits in the brain. Annu. Rev. Neurosci. 23, 155-184

Leonardo, E.D., Hen, R., 2008. Anxiety as a developmental disorder. Neuropsychopharmacology 33, 134-140.

Lindhorst, T., Beadnell, B., Jackson, L. ., Fieland, K, Lee, A., 2009. Mediating pathways explaining psychosocial functioning and revictimization as sequelae of parental violence among adolescent mothers. Am J Orthopsychiatry 79, 181-190

Lupien. S.]. McEwen, B.S., Gunnar, M.R., Heim, C., 2009. Effects of stress throughout the lifespan on the brain, behaviour and cognition. Nat. Rev. Neurosci. 10, 434-445.

Maier-Diewald, W., Wittchen, H.U. Hecht, H., Werner-Eilert, K, 1983. Die Mïnchner Ereignisliste (MEL). Anwendungsmanual. Max-Planck-Institut für Psychiatrie, München.

Matz, K., Pietrek, C, Rockstroh, B., in press. Stress in der Kindheit sensitiviert für Stress im Erwachsenenalter-eine Studie mit psychiatrischen Patienten. Z. Kl. Psych. Psychoth.

McEwen, B.S., 2003. Early life influences on life-long patterns of behavior and health. Ment. Retard. Dev. Disabil. Res. Rev. 9, 149-154.

Meyer, S.E. Chrousos, G.P., Gold. P.W., 2001. Major depression and the stress system: a life span perspective. Dev. Psychopathol. 13, 565-580.

Moratti, S. Rubio, G. Campo P. Keil A Ortiz, T, 2008. Hypofunction of right temporoparietal cortex during emotional arousal in depression. Arch. Gen. Psychiatr. 65, 532-541.

Morey, R.A., Petty, CM., Cooper, D.A., Labar, K.S., McCarthy, G., 2008. Neural systems for executive and emotional processing are modulated by symptoms of postraumatic stress disorder in Iraq War veterans. Psychiatr. Res. 162, 59-72

Mueser, KT. Penn, D.L. Blanchard, JJ. Bellack, A.S., 1997. Affect recognition in schizophrenia: a synthesis of indings across three studies. Psychiatr. Interpers. Biol. Process. 60, 301-308.

Nemeroff, CB., 2004. Neurobiological consequences of childhood trauma. J. Clin. Psychiatr. 65, 18-28.

Oldfield, R.C, 1970. The assessment and analysis of handedness: The Edinburgh inventory. Neuropsychologia 9, 97-113.

Pace, T.W. Mletzko, T.C. Alagbe, O., Musselman. D.L., Nemeroff, C.B., Miller, A.H. Heim, C.M., 2006. Increased stress-induced inflammatory responses in male patients with major depression and increased early life stress. Am. J. Psychiatr. 163, 1630-1633.

Peyk, P. Schupp, H.T., Elbert, T., Junghöfer, M., 2008. Emotion processing in the visual brain: a MEG analysis. Brain Topogr. 20, 205-215.

Peyk, P., Schupp, H.T., Keil, A, Elbert, T., Junghöfer, M., 2009. Parallel processing of affective visual stimuli. Psychophysiology 46, 200-208

Phan, K.L., Fitzgerald, DA., Gao, K., Moore, G.J., Tancer, M.E., Posse, S., 2004. Real-time fMRI of cortico-limbic brain activity during emotional processing. NeuroReport 15, 527-532.

Plotsky, P.M., Thrivikraman, KV., Nemeroff, CB., Caldji. C., Sharma, S., Meaney, M.]., 2005. Long-term consequences of neonatal rearing on central corticotropinreleasing factor systems in adult male rat offspring. Neuropsychopharmacology 30 , 2192-2204.

Pole, N., Neylan. T.C, Otte, C. Metzler, T.J., Best, S.R., Henn-Haase, C, Marmar, C., 2007. Associations between childhood trauma and emotion-modulated psychophysiological responses to starting sounds: a study of police cadets. J. Abnorm. Psychol. $116,352-361$.

Rockstroh, B., Junghöfer, M. Elbert, T., Buodo, G., Miller, G.A., 2006. Electromagnetic brain activity evoked by affective stimuli in schizophrenia. Psychophysiology 43, 431-439.

Sabatinelli, D., Flaisch. T., Bradley, M.M., Fitzsimmons. J.R. Lang, P.J., 2004. Affective picture perception: gender differences in visual cortex? NeuroReport 15 . 1109-1112.

Schupp, H.T. Junghöfer, M., Weike, A.l., Hamm, A.O., 2003. Emotional facilitation of sensory processing in the visual cortex. Psychol. Sci. 14, 7-13.

Schupp. H.T., Junghöfer, M., Weike, A.I., Hamm, A.O., 2004. The selective processing of briefly presented affective pictures: an ERP analysis. Psychophysiology 41, $441-449$

Schupp, H.T., Flaisch, T., Stockburger, J., Junghöfer, M., 2006. Emotion and attention: event-related brain potential studies. Progr. Brain Res. 156, 31-51.

Spinelli, S., Chefer, S., Suromi, S.J. Higley, J.D., Barr, C.S., Stein, E., 2009. Early-life stress induces long-term morphologic changes in primate brain. Arch. Gen. Psychiatr. 66 , $658-665$

Sterlemann, V., Ganea, K., Liebl, C., Harbich, D., Alam, S., Holsboer, F., Muller, M.B. Schmidt, M.V., 2008. Long-term behavioral and neuroendocrine alterations following chronic social stress in mice: implications for stress-related disorders. Horm. Behav. 53, 386-394.

Taylor, S.E., Eisenberger, N.I., Saxbe, D., Lehman, B.J., Lieberman, M.D., 2006. Neural responses to emotional stimuli are associated with childhood family stress. Biol. Psychiatr. 60, 296-301.

Tomoda, A., Navalta, C.., Polcari, A., Sadato, N., Teicher, M.H., 2009. Childhood sexual abuse is associated with reduced gray matter volume in visual cortex of young women. Biol. Psychiatr. 66, 642-648. 
Turkheimer, E., 2000. Three laws of behavior genetics and what they mean. Curr. Dir. Psychol Sci. 9, 160-164.

Van den Bergh, B.R. Van Calster, B., Smits, T., Van Huffel, S., Lagae, L, 2008. Antenata maternal anxiety is related to HPA-axis dysregulation and self-reported depressive symptoms in adolescence: a prospective study on the fetal origins of depressed mood. Neuropsychopharmacology 33, 536-545.

Walker, E., Mittal, V., Tessner, K., 2008. Stress and the hypothalamic pituitary adrenal axis in the developmental course of schizophrenia. Annu. Rev. Clin. Psychol. 4 , 189-216.
Weber, K., Rockstroh, B., Borgelt, ]., Awiszus, B., Popov, T., Hoffmann, K., Schonauer, K. Watzl, H., Pröpster, K, 2008. Stress load during childhood affects psychopathology Watzl, H., Pröpster, K, 2008. Stress load during
in psychiatric patients. BMC Psychiatry $8,63$.

Weber, K., Miller, G.A., Schupp, H.T., Borgelt, J., Awiszus, B., Popov, T., Elbert, T. Rockstroh, B., 2009. Early life stress and psychiatric disorder modulate cortical responses to affective stimuli. Psychophysiology 46, 1-10.

Wright M.O, Crawford, E, Del Castillo, D. 2009. Childhood emotional maltreatmen and later psychological distress among college students: the mediating role of maladaptive schemas. Child Abuse Neg1. 33, 59-68. 